\title{
Ballerina - pirouettes in search of gamma bursts
}

\author{
S. Brandt ${ }^{1}$, N. Lund ${ }^{1}$, H. Pedersen ${ }^{2}$, and J. Hjorth ${ }^{2}$ for the Ballerina Collaboration ${ }^{\star}$ \\ 1 Danish Space Research Institute, Juliane Maries Vej 30, DK-2100 Copenhagen $\varnothing$, Denmark \\ 2 Astronomical Observatory, University of Copenhagen, Juliane Maries Vej 30, DK-2100 Copenhagen $\varnothing$, Denmark
}

Received December 29, 1998; accepted May 4, 1999

\begin{abstract}
The cosmological origin of gamma ray bursts has now been established with reasonable certainty. Many more bursts will need to be studied to establish the typical distance scale, and to map out the large diversity in properties which have been indicated by the first handful of events. We are proposing Ballerina, a small satellite to provide accurate positions and new data on the gammaray bursts. We anticipate a detection rate an order of magnitude larger than obtained from Beppo-SAX.
\end{abstract}

Key words: gamma-rays: bursts — space vehicles

\section{Introduction}

The crucial observation of an X-ray transient following a cosmic gamma ray burst (GRB) was made by the Italian-Dutch satellite Beppo-SAX (Costa et al. 1997). Several GRBs with X-ray and optical afterglows have subsequently been found. In several cases, it has been possible to identify a remote galaxy as a likely host to the burst source, and to derive its distance. The huge luminosities, and the very short time scales involved in GRBs, may possibly be explained by merging, or collapsing, compact objects such as neutron stars or black holes (e.g. Paczyński 1986). However, the observed energy spectra of the bursts, and the details of the energy transport and conversion, are still to be explained. Models involving relativistic fireballs and jets have been proposed (e.g. Dar 1998). To date no theory has been able to explain all of the observed GRB phenomena, and it has become a challenge for modern astrophysics.

Send offprint requests to: N. Lund

* The Ballerina Collaboration presently consist of members from: Danish Space Research Institute; Astronomical Observatory, University of Copenhagen; Institute of Physics and Astronomy, Aarhus University; Aalborg University; Stockholm Observatory; Helsinki Observatory; University of Birmingham; Max Planck für Extraterrestische Physik, Garching; LAEFF, INTA, Madrid; Astronomiches Institut Potsdam.

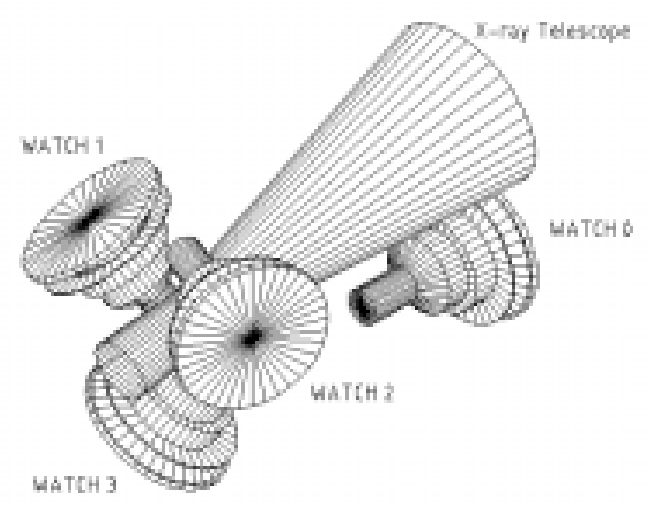

Fig. 1. A possible accommodation of the Ballerina payload with four WATCH wide field monitors and a grazing incidence X-ray telescope

\section{The Ballerina mission}

It remains an objective to ensure the earliest detection of the X-ray afterglow. Ballerina will for the first time allow systematic studies of the soft X-ray emission in the time interval from only a few minutes after the onset of the burst to a few hours later. Ballerina will, on its own provide observations in an uncharted region of parameter space. Positions of GRB sources with accuracy better than $1^{\prime}$ will be distributed within a few minutes of the burst.

Secondary objectives of the Ballerina mission includes observations of the earliest phases of the outbursts of $\mathrm{X}$-ray novae and other X-ray transients.

In addition to the autonomous observations of events detected on-board, Ballerina may on short notice be commanded from the ground to execute observations on objects identified by other observatories.

Ballerina is a spacecraft in the $100 \mathrm{~kg}$ class. The payload consists of an all-sky monitor and a grazing incidence X-ray telescope. A compact accommodation of these key elements is shown in Fig. 1. Ballerina is one of four missions currently under study for the Danish Small Satellite Programme. If selected, Ballerina will be launched in 2002. 


\subsection{The all-sky monitor}

The all-sky monitor consists of four Rotation Modulation Collimator instruments, arranged in a tetrahedron configuration to cover the full sky. Each unit is similar to the WATCH instruments flown successfully on the EURECA and GRANAT missions (Lund 1985; Castro-Tirado 1994; Sazonov et al. 1998; Brandt 1994). The detector used is a mosaic of $\mathrm{NaI}$ and CsI scintillators, with an energy range of $6-120 \mathrm{keV}$. The established threshold sensitivity of WATCH for a GRB of 5 second duration is $10^{-5} \mathrm{erg} / \mathrm{cm}^{2}$. Bursts of this fluence can be located to better than $1^{\circ}$ diameter $(2 \sigma)$.

Placed in a high (Molniya-type) orbit with an efficiency of $55 \%$, Ballerina will observe $\approx 80$ bursts per year distributed over the full sky. About 10 of these will be too close to the Sun to be observed with the X-ray telescope, but the remaining 70 bursts will be located to better than $0.5^{\prime}$.

The capacity of the telemetry link for Ballerina will be significantly better than available on GRANAT and on EURECA. We do not expect the telemetry to be a limiting factor for transmitting the data from the observed GRBs.

Efficient rejection of false triggers will be a high priority objective for the on-board software. The decision to slew the satellite to a new pointing will only be taken when the existence of a GRB (or X-ray transient) source has been confirmed by localizing the source consistently using two independent datasets. This is very efficient in rejecting false triggers. However, the real problem will be to manage the computational effort to search for two consistent localizations in the many data set combinations possible from a marginal trigger.

Satellite attitude slews will be executed solely by controlling the speed of each of the four WATCH modulators. Magneto-torquers will be used for momentum dumping.

\subsection{The grazing incidence $X$-ray telescope}

This instrument will be a smaller version of the instrument used on ROSAT with a focal length of $60 \mathrm{~cm}$ equipped with a CCD focal plane detector. Our design is based on a telescope with nested mirror shells, the required effective area being $50 \mathrm{~cm}^{2}$. The field of view is $1.5^{\circ}$, and the mirror shape will be optimized for achieving a rms resolution $<30^{\prime \prime}$ over the entire field. The mirror fabrication technique is similar to the one, which has been used with great success for the mirrors for Beppo-SAX and for ESA's XMM mission. The energy response will be in the range $0.5-2 \mathrm{keV}$.

The sensitivity of the Ballerina telescope will not be much different from the sensitivity of the Beppo-SAX telescopes. Consequently, it should be possible to follow the afterglows for $24-48$ hours. In fact, the bursts detected by the Ballerina all-sky monitor will on average be brighter, than the bursts seen by the SAX Wide Field Cameras. Therefore, we expect that also the afterglows will be brighter, and may be followed for a longer time. The orbit of Ballerina will permit up to 7 hours of uninterrupted observations of an afterglow source. This will allow to detect possible deviations from the simple power law decay of the afterglow.

\subsection{Burst operations sequence}

The satellite normally operates in a three-axis stabilized mode, performing survey observations and follow-up on previously detected bursts, waiting for a new burst to occur. The GRB is detected by one of the four wide-field cameras. An initial position with accuracy better than $1^{\circ}$ is derived, and transmitted to the ground. A slew is initiated to acquire the afterglow with the pointed X-ray telescope. The satellite will be able to slew to the new target in $20-70 \mathrm{~s}$, depending on the distance of the slew. A fine burst position (better than $30^{\prime \prime}$ ) is then determined.

The observations of the decaying afterglow are automatically scheduled to take spacecraft constraints into consideration. The full sky is accessible, except a cone around the Sun of $45^{\circ}$ half angle. Real time communication (at a low data rate) is continuously available. Source position information will be downlinked and distributed to the community as soon as it is available.

Modifications to the observation plan may also be uploaded from the ground.

\section{Conclusion}

Cosmic gamma ray bursts is a continuing enigma for astrophysics. We are proposing Ballerina, a small satellite to provide accurate GRB positions at a rate an order of magnitude larger than Beppo-SAX. The Ballerina spectral data will place powerful constraints on the theoretical work being done to understand GRBs. Establishing the cosmological distances to GRBs, and understanding their nature will provide us with a new and independent probe of the structure of the Universe - complementary to supernovae, quasars and clusters of galaxies.

Acknowledgements. Part of this work was supported by the Danish Space Advisory Board.

\section{References}

Brandt S., 1994, PhD. thesis, DSRI

Castro-Tirado A.J., 1994, PhD. thesis, DSRI

Costa E., et al., 1997, Nat 387, 876

Dar A., 1998, ApJ 500, L93

Lund N., 1985, SPIE Conf. Proc. 597, 95

Paczyński B., 1986, ApJ 308, L43

Sazonov Y., et al., 1998, A\&AS 129, 1 